\title{
RESEÑA
}

\section{MORAL Y ORDEN. SENTIDOS Y PRÁCTICAS EN LA TRANSFORMACIÓN DE LOS COMPORTAMIENTOS PÚBLICOS}

\author{
MORAL E ORDEM. SENTIDOS E PRÁTICAS NA TRANSFORMAÇÃO DE \\ COMPORTAMENTOS PÚBLICOS
}

\section{MORAL AND ORDER. SENSES AND PRACTICES IN TRANSFORMING PUBLIC BEHAVIORS}

Jorgelina Beltramone Universidad Nacional del Litoral (UNL)

RESEÑA DE SEDRÁN PAULA, MORAL Y ORDEN. SENTIDOS Y PRÁCTICAS EN LA TRANSFORMACIÓN DE LOS COMPORTAMIENTOS PÚBLICOS (SANTA FE, 18561890), BUENOS AIRES, TESEO, 2018, PÁG. 275.

Paula Sedrán, se ha ocupado de la denominada cuestión del orden en el espacio santafecino de fines del siglo XIX. Este recorte temático es fruto de diferentes elecciones, interrogantes y miradas, e implicó ubicar ciertos procesos socio-históricos locales, en el marco del proceso de formación del Estado-Nación. Tal como dice la autora, por esta vía consiguió "poner en cuarentena" muchas de las explicaciones establecidas, que se basan en los arraigados enfoques economicistas y políticos que solo ven en este periodo la conformación del sistema capitalista, y del Estado nación.

De este modo, logra acercarse a un espacio de vacancia en los estudios de este periodo, sobre todo desde nuevos interrogantes y latitudes aun no exploradas.

\begin{tabular}{|l|l|l|l|l|l|} 
Revista RBBA & ISSN 2316-1205 & Vitória da Conquista & V.9 $\mathrm{n}^{\circ} 1$ & p. 381-385 & Julho/2020
\end{tabular}




\section{MORAL Y ORDEN. SENTIDOS Y PRÁCTICAS EN LA TRANSFORMACIÓN DE}

LOS COMPORTAMIENTOS PÚBLICOS

A lo largo del libro el foco está puesto en el reordenamiento de las relaciones sociales en la ciudad, en torno a lo que se conoció como la cuestión del orden y los diferentes mecanismos estatales de control de los comportamientos en los espacios públicos que se implementaron en Santa Fe entre los años 1856 y 1890. Para ello, la autora se centra en dos cuestiones estrechamente relacionadas siendo los procesos confluyentes de la práctica y la simbolización de la moral.

En primer lugar, se ocupa de posicionarse en el campo, y referenciar desde que perspectiva emprende su estudio. Toma como referencia la historia social y cultural, y subcampos dedicados al castigo y al delito. Para ello, acude a la idea de un "terreno contestado" de Salvatore, ya que en cada contexto especifico los sentidos del orden, delito y violencia se dirimieron de diversa manera. Los nuevos enfoques del delito focalizan no solo la imposición del derecho legal, sino las interacciones con los agentes en cuestión. El análisis de los discursos policiales y estatales, permiten adentrarse a las representaciones y concepciones sobre el orden deseable. Y en esta línea, este terreno contestado del orden fue más allá e incluyó discursos literarios, periodísticos y de asociaciones civiles. Esta delimitación de un horizonte moral deseable, sirve de base para analizar las prácticas de control concretas y estables en la ciudad, institucionalizando pautas de comportamiento legítimas y aquellas que debían eliminar del espacio público.

Otra vertiente de la que se nutre son aquellas investigaciones centradas en la construcción de límites simbólicos en las sociedades de la modernidad, y las problemáticas de la identidad y construcción de "otros internos". Y en correlación con ello, ciertos estudios que focalizan en las prácticas concretas de la estigmatización social que delimitaron lo moral e inmoral y a partir de ello los criterios de inclusión y exclusión en la concepción de la sociedad.

Sedrán trabaja desde la perspectiva de la historia regional. En este camino, el objeto de análisis y la región se definen relacionalmente, y el enfoque regional se instaura como un modelo explicativo permitiendo adecuar la escala con la que se aborda una problemática. Antes de tomar la ciudad como objeto en este trabajo son los "lugares" de la ciudad en los que se visibilizaron ciertos comportamientos y a partir de ello avanzar en explicaciones situadas.

En el primer capítulo, se ocupa de la construcción de representaciones sociales en torno a la cuestión del orden. La dimensión simbólica, es un punto de arranque para acercarse a las prácticas de control de las transgresiones y las concepciones sobre la violencia dentro de la \begin{tabular}{l|l} 
Revista RBBA & Revista Binacional Brasil Argentina
\end{tabular} 


\section{MORAL Y ORDEN. SENTIDOS Y PRÁCTICAS EN LA TRANSFORMACIÓN DE}

LOS COMPORTAMIENTOS PÚBLICOS

ciudad. A partir del análisis de los discursos de las elites, ya sea aquellos expresados desde el poder ejecutivo provincial como así también aquellos esbozados desde las dependencias de la policía (ambas en proceso de institucionalización), la historiadora advierte que "la moral dio contenido al orden". La moral fue una variable que incidió de manera decisiva en la delimitación de un nuevo orden.

En el marco de la conformación del Estado y su materialización en lógicas institucionales concretas, las representaciones sociales sobre el orden deseable se establecieron como un pivoteo de la dominación simbólica.

Sedrán a lo largo de su trabajo, parte de las fuentes escritas disponibles, es decir documentos institucionales (informes policiales, y del ejecutivo provincial) o documentos no institucionales como la prensa. Desde esta perspectiva, contempla una ausencia de las voces de los sectores populares ya que la producción de los documentos es resultado de "procesos hegemónicos" y los documentos analizados permiten visualizar las voces de las clases dominantes de la época. Tales discursos de las elites del periodo, definieron un orden deseable, la violencia y la otredad, y estuvieron estrechamente vinculadas con sus creencias, proyectos, filiaciones políticas, objetivos económicos.

Las clases dominantes construyeron la otredad en base a la simbolización de la violencia desde el eje de la moralidad. Asi, la violencia y los violentos son representados como lo inmoral, lo incontrolable. Al respecto, Sedrán precisa que "la violencia que amenaza el normal decurso de la vida social, está compuesta por aquellos afectos, pasiones, emociones que no pertenecen a lo que la sociedad en su conjunto ha aceptado como tolerable" (SEDRÁN, 2018, p. 35).

El segundo capítulo, focaliza en los sentidos construidos desde la prensa, y los discursos asociativos o literarios acerca de la cuestión del orden y los sujetos transgresores e inmorales. Estos discursos de la sociedad civil, permiten complementar el enfoque anterior, en torno a la visión de las clases dominantes. Asociaciones como las Damas de Beneficencia, que en esos años estaban cumpliendo funciones de caridad y servicios sociales, construían la imagen de un "otro" social, en torno a los "desgraciados", los más "necesitados" y víctimas de los "dolores" e "infortunios" quienes por ello "precisan el auxilio de su caridad" (SEDRÁN, 2018, p. 87). En dichos discursos el sujeto se define como "sujeto de cuidado", ya que necesitaban de la asistencia de estas instituciones. Por medio de estos discursos cargados de sentido religioso, buscaban reafirmar su rol en la sociedad, solucionando problemas de orden y moral en la sociedad santafecina.

\begin{tabular}{l|l} 
Revista RBBA & Revista Binacional Brasil Argentina
\end{tabular} 


\section{MORAL Y ORDEN. SENTIDOS Y PRÁCTICAS EN LA TRANSFORMACIÓN DE}

LOS COMPORTAMIENTOS PÚBLICOS

Por otro lado, la autora analiza producciones culturales de la elite como crónicas, ensayos, noticias, y otros géneros, en donde el reclamo por los valores civilizatorios es frecuente. Lo que predomina es la construcción de una identidad cultural de las elites criollas con modelos europeos, remarcando la falta de moral de las costumbres de las clases populares que debían ser modificadas para que la sociedad santafecina sea verdaderamente civilizada.

Además, la prensa participó de diversas formas en la construcción de sentidos sobre la violencia y sobre la necesidad de un orden. El sector opositor, en un contexto de enfrentamientos facciosos de las elites políticas, denunciaba la falta de moralidad de la tropa policial, ya que los mismos que eran detenidos por cometer actos inmorales, eran los que debían custodiar el orden público. Es así, que la unión de la cuestión del orden y las contiendas políticas fue muy común en estos años.

En el tercer capítulo, repone la dinámica complementaria de la construcción de un orden. Hasta el momento, se acudió a una reconstrucción de las representaciones sobre que conductas son toleradas, y cuáles son las rechazadas y penadas por una sociedad que anhela la civilización; y quienes eran los sujetos sociales que eran inherentemente peligrosos para ese orden deseable. A partir de ello, Sedrán se ubica en el cruce entre las normativas policiales y las prácticas concretas de control del espacio público. Es decir, los mecanismos de imposición de un orden social, esto es, la dinámica material de "la imposición de un orden burgués en las calles santafecinas" (SEDRÁN, 2018, p. 141). Luego de un análisis de las faltas registradas por la policía, se concluye que hubo en el periodo ciertos comportamientos que fueron controlados y sancionados de manera estable, y otros que según la coyuntura precisa tuvieron picos de importancia. En este sentido, las causas más numerosas fueron por un lado la ebriedad, la pendencia y el escándalo, que infringían la respetabilidad de la institución policial y su imagen ante la sociedad. Y por otro lado, por ejemplo la portación de armas prohibidas o la falta de papeleta de enrolamiento iban en aumento en momentos de necesidad de la mano de obra en la institución policial.

La intersección entre representaciones y prácticas de control, da cuenta del carácter transicional de la sociedad santafecina en estos años. Por ello, se trató de una "institución conflictiva" la de la policía, ya que estaba caracterizada por una normativa precaria, unos recursos materiales y estructura institucional exigua y una composición social polarizada (SEDRÁN, 2018, p.158). 


\section{MORAL Y ORDEN. SENTIDOS Y PRÁCTICAS EN LA TRANSFORMACIÓN DE}

LOS COMPORTAMIENTOS PÚBLICOS

En el cuarto capítulo, analiza específicamente dos infracciones al orden que se volvieron preponderantes en este periodo. Sin descartar que en los documentos aparecen mencionadas diversas conductas consideradas inmorales y causa de violencia, fueron la ebriedad y el juego los vicios más mencionados y foco de mayores preocupaciones por parte de la policía. Se logra desandar una directa preocupación de la policía por el control de estos dos vicios considerados la causa de la inmoralidad y un "peligro moral para toda la sociedad", un flagelo. Las prácticas de control de la "moralidad" al interior de la policía fueron muy frecuentes en este periodo, y el análisis de ciertos sumarios específicos nos adentra en las tensiones en la institución policial, y como una forma de reafirmar la autoridad de control del orden deseado.

El libro hasta aquí analizado, es muy valioso para visualizar las prácticas y representaciones en torno al orden en el espacio santafecino, en un contexto de institucionalización del Estado Nación y de instauración de relaciones sociales capitalistas. Se remarca la necesidad de que el análisis sea proseguido frente a la disponibilidad de documentos, y lo fructífero que sería un análisis comparativo de este caso concreto con otros espacios del país.

\section{SOBRE LA AUTORA DE LA RESEÑA}

Jorgelina Sofia Beltramone es profesora de Historia por la Facultad de Humanidades y Ciencias de la Universidad Nacional del Litoral. Se especializo en los temas de la Historia Reciente Argentina, sobre todo la última dictadura cívico-militar, y la Guerra de Malvinas. Actualmente está realizando su tesis de grado para la Licenciatura en la misma casa de estudios.

Dirección electrónica: jorgibeltramone_06@hotmail.com

Orcid: https://orcid.org/0000-0001-6730-8761

Recebido em: 26/04/2020

Aprovado em: 05/06/2020

Publicado em: 01/07/2020 